\title{
Cell shape recognition and segmentation in fluorescence microscopy images
}

\author{
Alexander Anielski, Eva K.B. Pfannes and Carsten Beta \\ Manuscript received on June 22, 2012 / accepted on October 1, 2012
}

\begin{abstract}
Many cellular processes involve the translocation of proteins from the cytosolic region to the cortex of the cell and vice versa. The dynamics of such processes is typically investigated by fluorescence imaging of GFP-labeled versions of these proteins. Quantitative analysis of the resulting fluorescence images requires image segmentation procedures that identify the cell within the image and subsequently divide the cell into a cytosolic and a cortical region to monitor the temporal evolution of the fluorescence signals within these parts of the cell separately. Here, we present an image segmentation protocol that we have developed for this type of data analysis. It consists of noise reduction, normalization, and thresholding steps to generate masks that define the cytosolic and the cortical regions of a cell. Based on these masks, the desired fluorescence signals can be extracted from the confocal microscopy images.
\end{abstract}

Keywords: fluorescence microscopy, image processing, Dictyostelium.

\section{INTRODUCTION}

The rapid progress of modern life science depends to a large extent on the use of advanced imaging techniques [1, 2]. In particular, the design of fluorescent markers that can be genetically encoded and expressed in vivo have enormously contributed to our understanding of subcellular processes. Together with the development of advanced fluorescence imaging techniques, like confocal laser-scanning microscopy, spinning-disk microscopy, or total internal reflection fluorescence (TIRF) microscopy [1], the green fluorescent protein (GFP) and its variants have established the basis for modern live cell imaging. The award of the Nobel Prize in Chemistry 2008 to Shimomura, Chalfie, and Tsien can be seen as the most widely recognized reference to this groundbreaking contribution. In addition, the advent of superresolution microscopy in recent years highlights that we have only seen the beginning of a technical revolution that is about to lead life science imaging beyond the diffraction limit of classical microscopy [3].
Many cellular processes show dynamic changes over time. In particular, the study of motile cells requires that image acquisition and processing adapt to an ongoing change in cell position and shape, so that each image of a time series has to be newly segmented. This is a key prerequisite to implement a quantitative analysis of the subcellular dynamics and distribution of fluorescent labels. For example, studies of the dynamics of the actin cytoskeleton are highly dependent on such techniques. While many researchers face these requirements and develop their own computer based tools to solve such problems, only very few of these algorithms have been made public in all detail. One of the commonly used programs that meet some of these requirements is QuimP (Quantitative Imaging for Membrane Proteins) [4]. QuimP is implemented as a set of plugins for ImageJ, one of the most widely used image processing tools [5]. QuimP uses an active contour to automatically detect the outline of a cell in order to quantify fluorescence signals along the cell membrane [6] and 
has recently been extended to correlate the cortical fluorescence signals with membrane movement, a typical problem in fluorescence microscopy of motile cells [7]. A similar problem has been addressed before based on morphodynamic profiling [8]. Here, we present an alternative, MATLAB-based tool to quantify cortical and cytosolic fluorescence signals in motile cells.

\section{MATERIALS AND METHODS}

\subsection{Cell culture}

Dictyostelium discoideum cells expressing myosin II-GFP and LimE $\Delta$-mRFP in an AX2 background were grown in $\mathrm{HL}-5$ medium (Formedium, Hunstanton, UK) at room temperature $\left(21 \pm 2^{\circ} \mathrm{C}\right)$ in the presence of $0.01 \mathrm{mg} / \mathrm{mL}$ G418 and $0.01 \mathrm{mg} / \mathrm{mL}$ Blasticidin selection markers (Applichem, Darmstadt, Germany). Stimulation experiments were performed in 8 well slides (ibidi $\mathrm{GmbH}$, Martinsried, Germany) with cGMP and cAMP (Sigma-Aldrich, Taufkirchen, Germany).

\subsection{Microscopy and image acquisition}

Images were recorded on an LSM 710 confocal laser scanning microscope (Carl Zeiss Microimaging, Jena, Germany). The green and red labels were excited at $488 \mathrm{~nm}$ (argon laser) and $561 \mathrm{~nm}$ (DPSS laser). Images were taken at a rate of one frame per second.

\subsection{Image processing}

Images were processed on a Linux workstation (OpenSUSE 11.3 64 bit) with an Intel Pentium T4400 CPU and 4 GB RAM. A custom-made MATLAB program (MATLAB R2008b, Mathworks, Ismaning, Germany) was used. The details of the image processing protocol are described below.

\section{IMAGE SEGMENTATION PROTOCOL}

In this section, we provide a detailed description of the segmentation procedure that we have developed to process fluorescence images of single amoeboid cells. In particular, this protocol was used to analyze dual color time lapse recordings obtained by laser-scanning confocal microscopy. The segmentation algorithm consists of three successive steps: noise reduction, normalization, and thresholding.

\subsection{Three-dimensional noise reduction}

The laser light typically used to excite GFP-derived fluorophores may cause considerable photo-damage in many live cell imaging applications. To reduce the harmful influence of the excita- tion light, its intensity and exposure time are reduced to the minimal possible values. This often results in noisy raw images that cannot be used directly for cell shape recognition. Thus, prior to image segmentation, the noise needs to be reduced. Typical strategies involve the application of blurring kernels that replace each pixel value by a local average of the pixel's surrounding. In this way, an increased continuity in brightness values and shape can be achieved. In order not to lose structural details of the image, the size of the blurring kernel has to be chosen large enough to efficiently reduce the noise on the pixel level but at the same time small enough so that structural features of the image are not erased.

The typical confocal microscopy images taken in the course of our live cell studies, have a resolution of $512 \times 512$ pixels, where 1 pixel correspond to $0.264 \mu \mathrm{m}$. They are usually recorded in time lapse sequences of 1 frame per second. In this case, a rectangular blurring kernel of $5 \times 5$ pixels has proven to be a good choice. Here, we would like to draw the attention to the point that noise reduction could be significantly improved, if not only the two-dimensional neighborhood within one time frame was taken into account, but the entire three-dimensional neighborhood involving also the neighboring pixels from the adjacent previous and following frames along the time coordinate. This three-dimensional blurring relies on a rectangular kernel of $5 \times 5 \times 5$ pixels, where the third dimension extends along the time coordinate.

To demonstrate the effect of blurring, we present fluorescence images of a Dictyostelium cell that carries two fluorescent markers, a red fluorescent protein (mRFP) fused to LimE $\Delta$ and a green fluorescent protein (GFP) tagged to myosin II. Both proteins are associated with the actin cytoskeleton. The LimE $\Delta$ protein is known to colocalize with filamentous actin and has been established as one of the most widely used labels for polymeric actin structures in the cell [9]. Myosin II, on the other hand, is a motor protein that mostly localizes at the back of migrating amoeboid cells, where it mediates contraction of the cell cortex [10]. In Figure 1, the red (left) and green channels (right) of a dual color confocal microscopy image are displayed, showing the subcelIular distributions of LimE $\Delta-\mathrm{mRFP}$ and myosin II-GFP, respectively. In the top row, the raw data is shown, whereas the same images after application of three-dimensional blurring can be seen in the bottom row.

To illustrate the differences between two-dimensional and three-dimensional blurring, we present another example in Figure 2. Here, the application of different two-dimensional and 

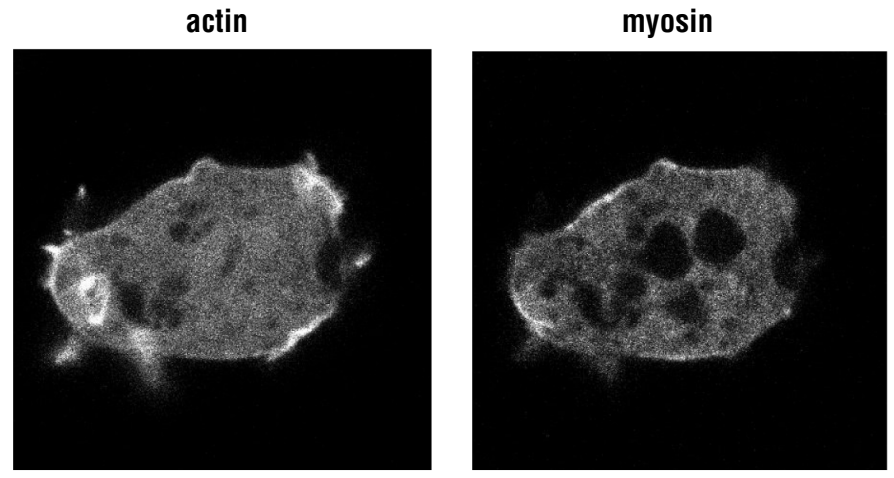

original pictures
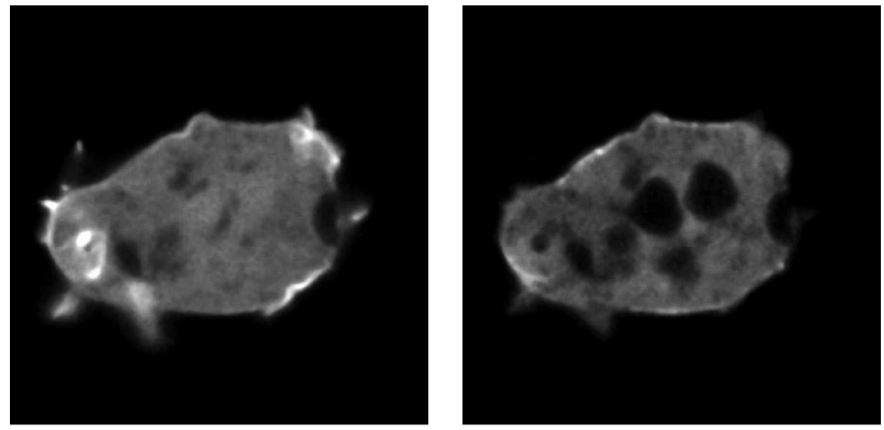

3D blurred pictures

Figure 1 - Example of the application of a 3D blurring kernel to a dual color confocal recording. The images show a Dictyostelium cell that expresses mRFP fused to $\operatorname{LimE} \Delta$, a marker for filamentous actin, and GFP fused to myosin II. The red channel (actin) is shown on the left, the green channel (myosin) on the right. The top row shows the origial raw images, whereas in the bottom row the same images after application of the 3D blurring kernel are displayed.
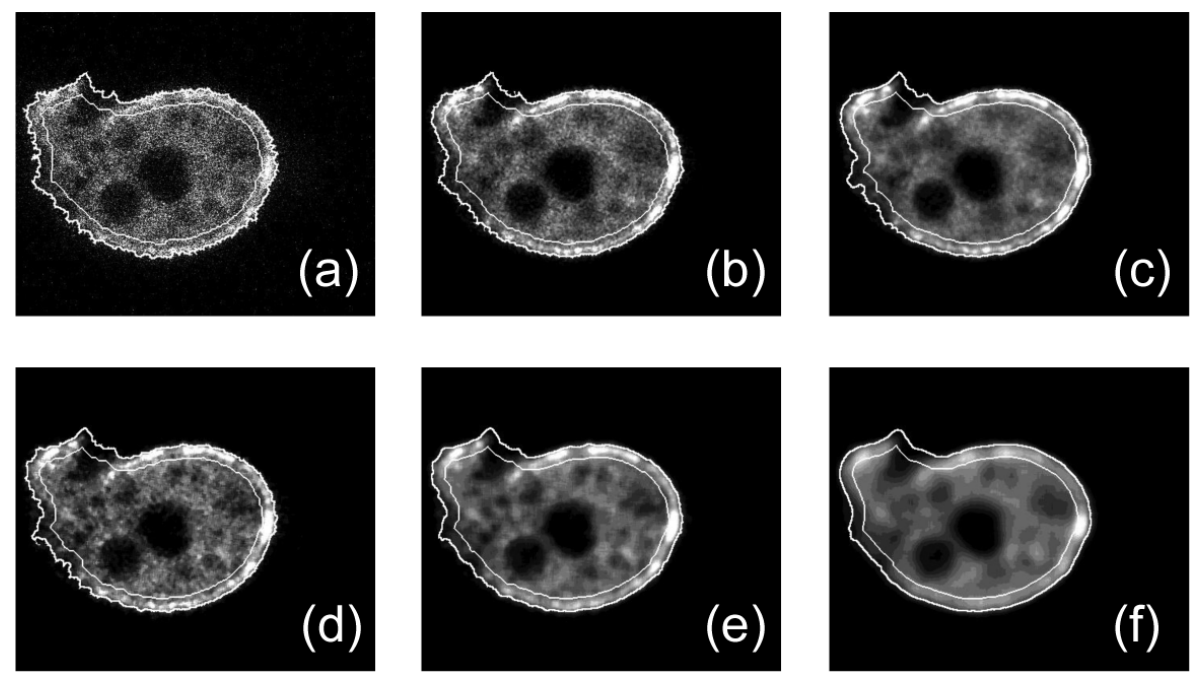

Figure 2 - Fluorescence image of a Dictyostelium cell expressing myosin II-GFP and LimE $\Delta$-mRFP. The myosin distribution is shown, where the raw data (a) is compared to the results of applying (b) $3 \times 3 \times 3$, (c) $5 \times 5 \times 5$, (d) $5 \times 5$, (e) $10 \times 10$, and (f) $20 \times 20$ blurring kernels. 
three-dimensional blurring kernels is compared. It can be clearly seen that three-dimensional blurring results in a smoother image without losing additional details as is the case for larger twodimensional kernels.

\subsection{Normalization}

Following noise reduction by three-dimensional blurring, the images are normalized. The overall sequence of operations involved in this process is summarized as a flow chart in Figure 3.

\section{Single frame (after 3D-blurring)}

Take subset of $1-5 \%$ randomly chosen pixels and arrange them as 1D vector

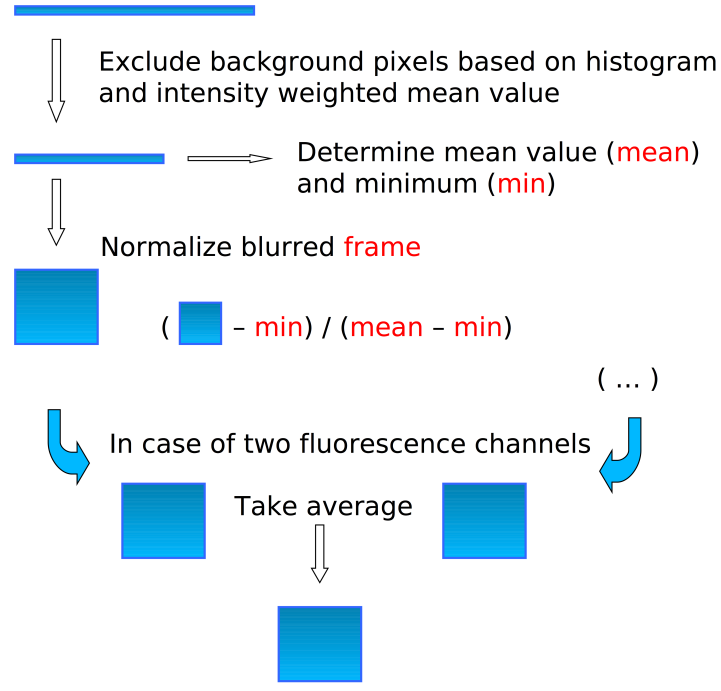

Figure 3 - Flow chart summarizing the individual steps involved in normalization of confocal microscopy images.

In order to reduce computational time, between 1 and $5 \%$ of the total number of pixels are randomly extracted from the image. From the intensity values of these pixels, a histogram is computed. If the number of extracted pixels is large enough, the histogram will resemble the intensity distribution of the total image. (Note that this has only a minor effect on the computation time. In cases where computational costs are of only secondary importance, the histogram can be also computed from the total number of pixels). In Figure 4 on top, an example for a normalized histogram is shown. It was generated from a single cell fluorescence image, similar to the images displayed in Figure 1. Such images are typically composed of a small number of bright pixels that belong to the area covered by the cell, and a larger number of dark pixels that are representing the background. The latter can be clearly identified as a maximum in the histogram at low inten- sity values. The smaller broad peak to the right of the background (towards larger intensities) can be attributed to the pixels inside the cell area.
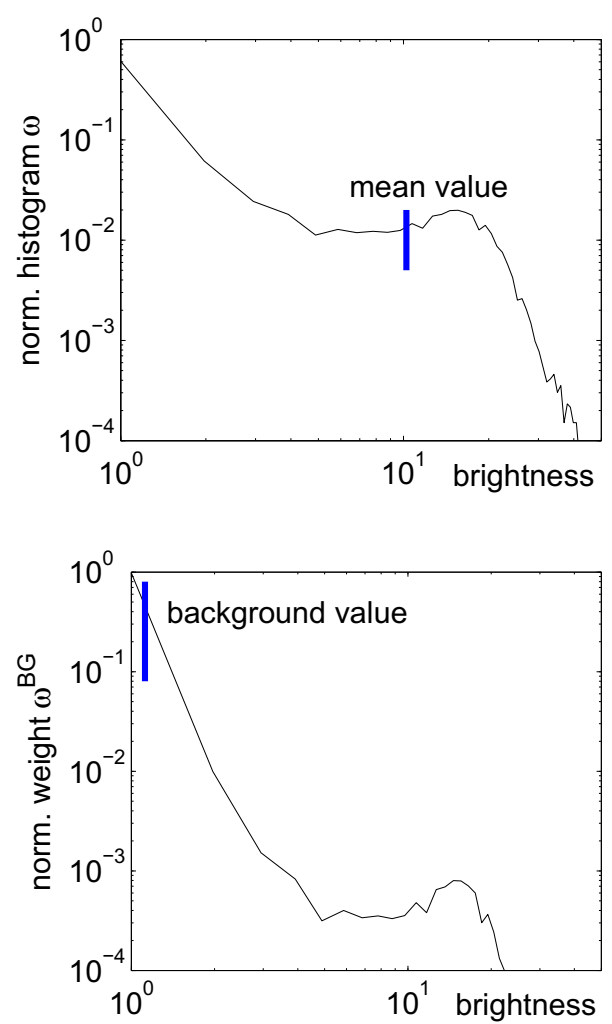

Figure 4 - (Top) Typical histogram (normalized) of a single cell fluorescence image, mean value marked by a vertical blue line. (Bottom) The same histogram as on the left, weighted with the weighting function (3) and normalized, mean value marked by a vertical blue line. Note that we have displayed intensity values ranging from 1 to 256 (instead of 0 to 255) in order to avoid problems with the logarithmic representation.

To remove the background pixels, a cutoff value has to be defined based on the histogram. The mean value,

$$
\langle f\rangle=\sum_{n=1}^{N} w_{n} f_{n},
$$

typically lies much higher than the background. It is computed from the range of possible intensity values $f_{n}$ and the normalized histogram

$$
w_{n}=\frac{h_{n}}{\sum_{n=1}^{N} h_{n}},
$$

where $h_{n}$ denotes the number of times that the intensity values $f_{n}$ occur in the image. To generate an appropriate cutoff value, we rescale the histogram prior to normalization with an empirical weighting function

$$
g_{n}=\left(f_{\max }-f_{n}\right) h_{n}^{2}
$$


that gives more statistical weight to the low-intensity background part and to those parts that show a large probabiliby. We thus obtain

$$
w_{n}^{B G}=\frac{g_{n}}{\sum_{n=1}^{N} g_{n}},
$$

which is then used in Eq. (1) instead of $w_{n}$. By adjusting the weighting function, the mean value taken over the weighted histogram can be shifted until it lies close to the maximum in the histogram at low intensity values, see Figure 4 , bottom. This shifted mean value is then used as a cutoff to remove the background pixels from the entire image. Its position close to the maximum was empirically found to be optimal for removing the background from the image. In the case shown here, only the lowest intensity value is removed by background subtraction. Note that for other cases, where the background shows a larger average intensity, our procedure works equally well. Once the weighting function is optimized to find the cutoff value, it can be used to automatically remove the background from an entire sequence of images that were recorded in the course of a live cell experiment.

From the remaining pixels that do not belong to the background, the minimum intensity and the mean value are determined. They are used to normalize the full image according to

$$
f_{i j}^{\text {norm }}=\frac{f_{i j}-\min \left(f_{i j}\right)}{\left\langle f_{i j}\right\rangle-\min \left(f_{i j}\right)},
$$

where $f_{i j}$ are the intensity values of the individual pixels of the image. In the case of two (or more) simultaneously recorded fluorescence channels, each image is normalized separately according to the procedure described above. For the subsequent thresholding and segmentation the normalized images from the different channels are averaged for each time step. Note that if the different channels show strongly different noise levels, the quality of the combined image can be degraded. In such cases, the noise reduction in each channel has to be adapted individually. This, however, was not the case for our data.

\subsection{Thresholding}

With the normalized image as a starting point, we now apply a threshold to identify the region of the cell. We then perform the actual segmentation to divide the cell area into cortical and cytosolic regions. The individual steps of this procedure are schematically listed in Figure 5 together with an example image that we show for each of the intermediate stages of this sequence of image processing operations.

First, we apply the MATLAB function imfill to eliminate dark areas inside the cell. They frequently occur as a consequence of inhomogeneities in the distribution of fluorescent markers that are caused by organelles and other intracellular structures. Then the actual thresholding is performed. All pixels of the normalized image that show intensity values larger than 0.4 are taken as part of the cell. They are set to the value of 1 . All remaining pixels shall belong to the background and are set to zero. In many cases, more than one cell can be found within the image. This will result in separate regions identified by the thresholding. Also, complex three-dimensional cell shapes may lead to several smaller disjunct regions when taking a single confocal cut through the cell. We apply the MATLAB function bwlabel to assign different integer numbers to these separate interconnected regions. The cell of interest is typically identified as the largest connected object among these regions. Alternatively, also the proximity of the regions to the center of the image may be employed as a criterion to select the cell area.

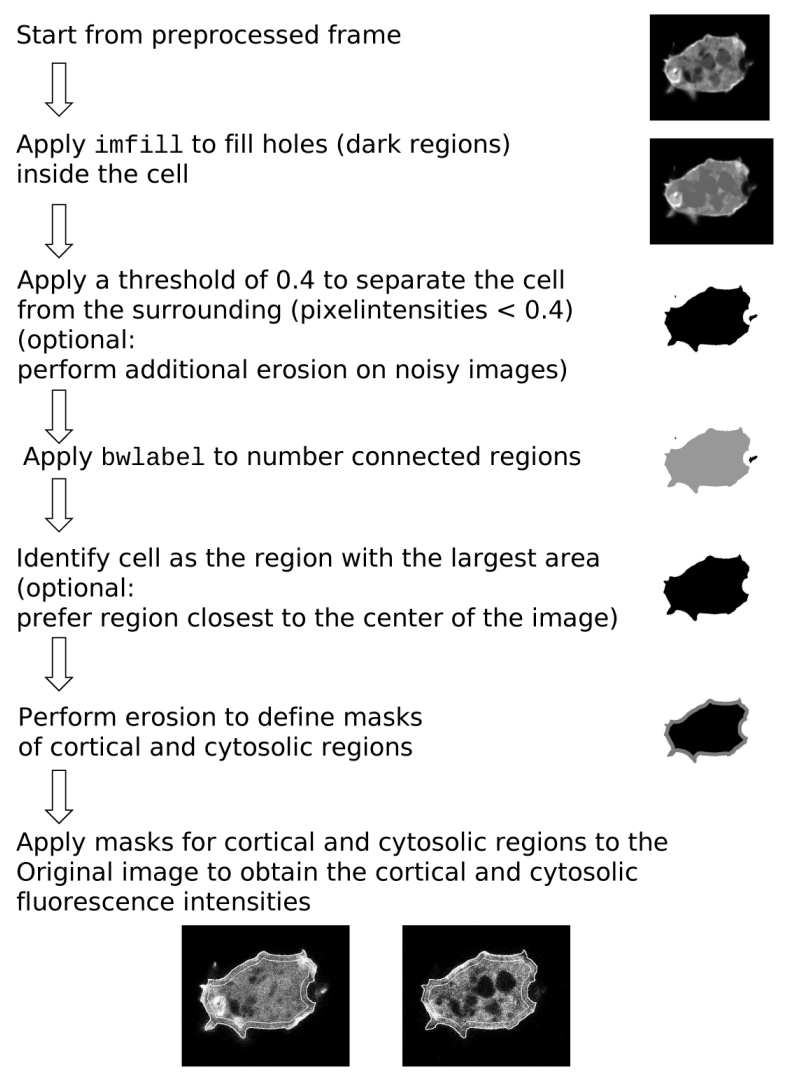

Figure $\mathbf{5}$ - Flow chart summarizing the individual steps involved in thresholding and segmentation of the cell into cortical and cytosolic regions.

Once the cell of interest is identified, its area is segmented into a cortical and a cytosolic part. This is achieved by eroding the cell area using the MATLAB function imerode. The remaining region is designated as the cytosolic part of the cell, whereas 
the eroded zone around the border is taken as the cell cortex. The size of the eroded zone can be adapted by modifying the structuring element that imerode uses for the erosion process, see also the MATLAB documentation [11]. The resulting masks for the cortical and cytsolic regions are multiplied with the original raw image to extract the intensity values of the pixels that belong to the respective parts of the cell. From these pixels, averaged cortical and cytosolic fluorescence intensities can be computed for each time step of a confocal recording.

\section{EXAMPLE}

Let us now turn to an example to illustrate the type of analysis that can be performed based on the image processing protocol described above. It has been reported that the CAMP receptors of Dictyostelium have a reduced affinity also to certain CAMP derivatives and other cyclic nucleotides [12, 13]. In particular, the affinity to cGMP is very low, so that an upshift in extracellular CGMP is not expected to trigger the typical intracellular receptor responses, when supplied at physiological concentration levels. To exemplify this fact, we have stimulated starvation-developed Dictyostelium cells that express myosin II-GFP and, as a marker for F-actin, also LimE $\Delta$-mRFP, with a sudden step-like increase in the extracellular concentration of cGMP. This was achieved by adding the cGMP with a pipette to the cell culture that was plated in an 8 well slide. The concentration of the cGMP solution was chosen such that the final concentration in the culture well was $10 \mu \mathrm{M}$. The dynamics of both LimE $\Delta$-mRFP and myosin II-GFP in response to the stimulus were simultaneously imaged by dual color laser scanning confocal microscopy. The resulting data was compared to cells of the same type that were exposed to a similar stimulus in the extracellular CAMP concentration to provide a reference case for a typical receptor response.

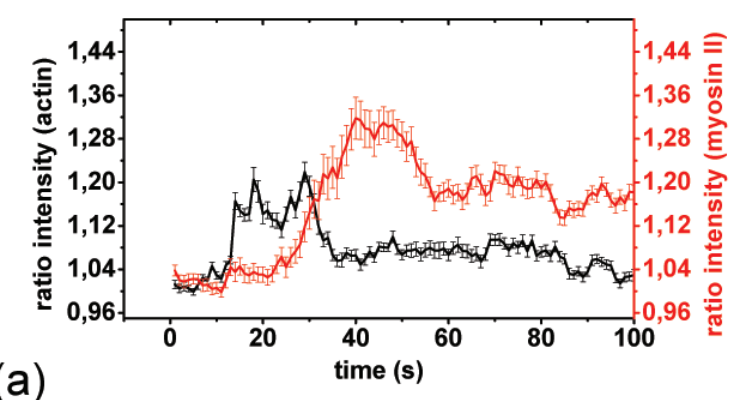

(a)

Figure 6 - Example of myosin II and actin dynamics in response to a stimulus with extracellular cAMP (a) and CGMP (b). The curves show the temporal evolution of the cortical divided by the cytosolic fluorescence intensities for the LimE $\Delta$-mRFP (black) and myosin II-GFP (red) labels, averaged over (a) 13 and (b) 10 single cell experiments. The error bars denote the standard error of the mean.
When exposed to an upshift in extracellular CAMP, starvationdeveloped Dictyostelium cells showed a boost in actin polymerization. This was reflected by the recruitment of LimE $\Delta$-mRFP to the cell cortex, accompanied by a reciprocal depletion of the cytosolic fluorescence intensity. Similarly, also myosin II accumulated in the cell cortex, following a cAMP receptor stimulus. In Figure 6(a), the actin and myosin II signals can be seen, averaged over 13 cells. The fluorescence images were processed according to the segmentation protocol described above, and the cortical divided by the cytosolic fluorescence intensities are displayed for both labels. The peak responses of both proteins were reached about $25 \mathrm{~s}$ apart. This is in agreement with earlier results, where a similar time shift between the actin and myosin responses has been reported [15]. Note, however, that our data is less precise than the results presented in [15] because we have dropped the cAMP solution into the culture well with the developed cells, whereas Etzrodt and coworkers have used a flow chamber in order to precisely control the timing of the chemical stimulus.

In Figure 6(b), we show the response of the same type of cells to a cGMP stimulus. Data averaged over 10 single cells is displayed. A comparison with the CAMP-stimulation data in Figure 6(a) clearly reveals that a comparable CGMP stimulus does not trigger the typical cytoskeletal response within the cell. Neither an early boost in actin polymerization nor the later accumulation of myosin II in the cell cortex were observed, when the cells were exposed to extracellular cGMP. This confirms the earlier biochemical results on the much lower receptor affinity of cGMP as compared to cAMP $[12,13]$.

\section{DISCUSSION}

In this article, we have presented a protocol for cell shape recognition and segmentation of fluorescence images taken by laser-

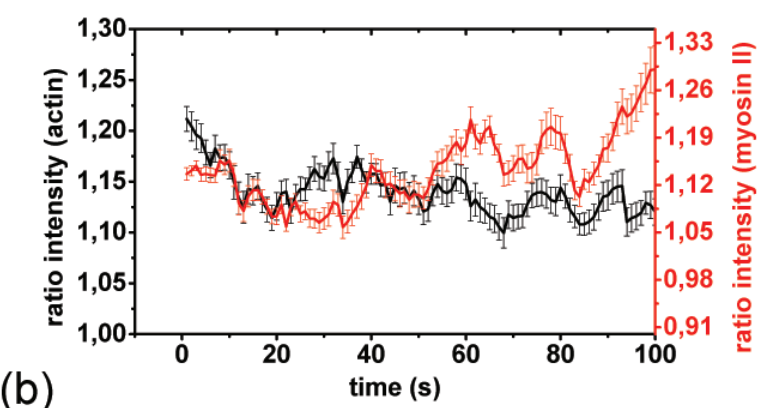


scanning confocal microscopy. Our algorithm involves three successive stages, namely noise reduction, normalization, and thresholding of the image. It is specifically tailored to analyze protein translocation events between the cytosolic and the cortical regions of motile cells. Note that the three-dimensional noise reduction, as proposed here, may run into problems if the cell moves considerably over the time of the five adjacent frames that form the third dimension of the 3D blurring kernel. If the speed of the imaging system is sufficient, this problem can be circumvented by increasing the imaging frame rate. However, this requires that the photo-damage per frame is low enough in order to prevent rapid degradation of the sample. Alternatively, the number of time frames that are incorporated into the blurring kernel could be reduced. Instead, noise in the individual frames could be additionally reduced by increasing the exposure time.

Our segmentation algorithm is based on a thresholding procedure that only indirectly uses information from the image histogram (the background subtraction prior to image normalization relies on the histogram). This is in contrast to other commonly used histogram-based segmentation methods like Otsu's method [16] or the Bi-Level Image Thresholding [17], where the threshold value is computed directly from a bi-modal image histogram. In our case, we often encountered distorted uni-modal histograms for which our method was found to give more reliable results as compared to methods optimized for clearly bimodal histograms. Alternative options are geometric active contour approaches like the Chan-Vese algorithm [18]. However, in the case of our fluorescence images that show a clearly defined cell (connected region of bright pixels) on a dark background, we obtained satisfactory results already with the simple global intensity threshold as described above.

We do not claim that the segmentation protocol described here is the only way to extract the desired information from the fluorescence images. Other approaches may yield similar results. In particular, there are software packages that show many userfriedly features like a graphical user interface (GUI) and compatibility with common image processing and graphics programs. A prominent example is the ImageJ plug-in QuimP, one of the most widely used tools for the processing of live-cell fluorescence images $[4,6]$. Table 1 shows a comparison between features of the QuimP software and our segmentation protocol. QuimP combines a sophisticated image segmentation approach with the analysis of other cell parameters (shape, motion etc.) and integrates these functionalities into a user-friendly GUI that is amenable to a wide public of users from the life science community. On the other hand, our segmentation protocol is comparatively simple and implemented in a short MATLAB script. It thus addresses the different needs of the computer-educated user that demands a starting point to write his own custom-made image processing software, which he can readily change, extend, and adapt to his specific problems.

Table 1 - Comparison between our segmentation protocol and the software package QuimP.

\begin{tabular}{|c|c|c|}
\hline & $\begin{array}{l}\text { Segmentation protocol } \\
\text { presented here }\end{array}$ & QuimP \\
\hline Programming language & MATLAB & Java \\
\hline Compatibility & any MATLAB program & ImageJ \\
\hline Degree of complexity & medium & high \\
\hline Flexibility & high & medium \\
\hline Cell detection & Intensity threshold & Active contour \\
\hline Resolution & temporal & spatiotemporal \\
\hline Correlation with movement & no & yes \\
\hline Graphical user interface & no & yes \\
\hline
\end{tabular}

We have demonstrated the function of our approach using cells of the social amoeba Dictyostelium discoideum, a popular model organism for the study of eukaryotic cell motility and chemotaxis. We used a construct that simultaneously expressed two fluorescent markers fused to proteins associated with the actin cytoskeleton. The temporal evolution of the cytosolic and cortical localization of these labels could be successfully followed over time to analyze the response of the cell to different extracellular ligands. We hope that our approach will make a useful contribution to the active and expanding area of live cell imaging applications at the interface of physics and biology.

\section{ACKNOWLEDGMENTS}

We gratefully acknowledge financial support by the Deutsche Forschungsgemeinschaft in the framework of the grant BE 3978/ 3-1.

\section{REFERENCES}

[1] GOLDMAN RD, SWEDLOW JR \& SPECTOR DL (Eds.). 2010. Live Cell Imaging. Cold Spring Harbor Laboratory Press.

[2] ISHIKAWA-ANKERHOLD HC, ANKERHOLD R \& DRUMMMEN GPC. 2012. Advanced Fluorescence Microscopy Techniques - FRAP, FLIP, FLAP, FRET and FLIM. Molecules, 17: 4047-4132.

[3] HELL SW. 2007. Far-Field Optical Nanoscopy. Science, 316: 1153-1158. 
[4] http://www2.warwick.ac.uk/fac/sci/systemsbiology/staff/bretschneider/ quimp/

[5] http://rsbweb.nih.gov/ij/

[6] DORMANN D, LIBOTTE T, WEIJER CJ \& BRETSCHNEIDER T. 2002. Simultaneous Quantification of Cell Motility and ProteinMembrane-Association Using Active Contours. Cell Motility and the Cytoskeleton, 52: 221-230.

[7] BOSGRAAF L, VAN HAASTERT PJM \& BRETSCHNEIDER T. 2009. Analysis of Cell Movement by Simultaneous Quantification of Local Membrane Displacement and Fluorescent Intensities Using Quimp2. Cell Motility and the Cytoskeleton, 66: 156-165.

[8] MACHACEK M \& DANUSER G. 2006. Morphodynamic Profiling of Protrusion Phenotypes. Biophysical Journal, 90: 1439-1452.

[9] SCHNEIDER N, WEBER I, FAIX J, PRASSER J, MÜLLER-TAUBENBERGER A, KÖHLER J, BURGHARDT E, GERISCH G \& MARRIOTT G. 2003. A Lim Protein Involved in the Progression of Cytokinesis and Regulation of the Mitotic Spindle. Cell Motility and the Cytoskeleton, 56: 130-139.

[10] YUMURA S \& FUKUI Y. 1985. Reversible cyclic AMP-dependent change in the distribution of myosin thick filaments in Dictyos telium. Nature, 314: 194-196.

[11] http://www.mathworks.de/help/techdoc/
[12] VAN HAASTERT PJM \& KIENE. 1983. Binding of CAMP Derivatives to Dictyostelium discoideum Cells. Journal of Biological Chemistry, 258: 9636-9642.

[13] JOHNSON RL, VAN HAASTERT PJM, KIMMEL AR, SAXE III CL, JASTORFF B \& DEVREOTES PN. 1992. The Cyclic Nucleotide Specificity of Three cAMP Receptors in Dictyostelium. Journal of Biological Chemistry, 267: 4600-4607.

[14] GOELDNER M \& GIVENS R (Eds.). 2005. Dynamic Studies in Biology: Phototriggers, Photoswitches an Caged Biomolecules. Wiley-VCH.

[15] ETZRODT M, ISHIKAWA HCF, DALOUS J, MÜLLER-TAUBENBERGER A, BRETSCHNEIDER T \& GERISCH G, 2006. Timeresolved responses to chemoattractant, characteristics of the front and tail of Dictyostelium cells. FEBS Letters, 580: 6707-6713.

[16] OTSU N. 1979. A Threshold Selection Method from Gray-Level Histograms. IEEE Transactions on Systems, Man, and Cybernetics, 9: 62-66.

[17] DOS ANJOS A \& SHAHBAZKIA HR. 2008. Bi-level image thresholding - A fast method. Biosignals 2008. Proceedings, 70-76.

[18] CHAN TF \& VESE LA. 2001. Active Contours Without Edges. IEEE Transactions on Image Processing, 10: 266-277. 